\title{
ON FINDING THE EHRHART POLYNOMIALS USING A MODIFIED PARTIAL FRACTION METHOD
}

\author{
Shatha Assaad Al-Najjar, Samaa. F. A. and Vian A. Al-Salehy \\ School of Applied Sciences , University of Technology .
}

\begin{abstract}
:
A wide variety of topics in pure and applied mathematics involve the problem of counting the number of lattice points inside a polytope. Perhaps the most famous special case is the theory of Ehrhart polynomials, which is the basis structure theorem about this type of counting problem.

We present a modified tool to find the Ehrhart polynomial of a convex polytope, by writing a polytope as a linear system and find the solution of this system using integer programming method with a modification on this method. This method depends on deriving the vector partition function as a partial fraction.
\end{abstract}

\section{Introduction}

We are interested in computing the number of integer solutions to the linear system $\mathrm{x} \in \mathrm{R}_{\geq 0}^{\mathrm{d}}$ (where $\mathrm{R}_{\geq 0}^{\mathrm{d}}$ means d-space of vectors with positive components), $A x=b$, where the coefficients of $A$ are non negative $(m \times d)$ integral matrix and $\mathrm{b} \in \mathrm{Z}^{\mathrm{m}}$.

Let $A$ fixed and study the number of solutions $\Phi_{\mathrm{A}}$ (b) as a function of $\mathrm{b}$, the function $\Phi_{\mathrm{A}}$ (b) often called a vector partition functions, which appears in mathematical areas: Number theory, Discrete Geometry, Commutative Algebra, Algebraic Geometry, Representation Theory, Optimization, as well as applications to Chemistry, Biology, Physics, Computer Science and Economics [1].

Denote the columns of A by $\mathrm{c}_{1}, \ldots, \mathrm{c}_{\mathrm{d}}$. For $\mathrm{P}$ a d-polytope, Ehrhart studied the particular case of $\Phi_{\mathrm{A}}$ (b) given by the counting function $\mathrm{L}(\mathrm{P}, \mathrm{t}) \#\left(\mathrm{tP} \cap \mathrm{Z}^{\mathrm{d}}\right)$, for positive integer $\mathrm{t}$, this number of lattice points in the dilation $\mathrm{tP}$ of $\mathrm{P}$. before we star, the following lemma is needed which go to Euler, [2].

\section{Properties of the Ehrhart polynomials:}

A convex polytope $\mathrm{P} \subset \mathrm{R}^{\mathrm{d}}$ is the convex hull of infinity many points in $\mathrm{R}^{\mathrm{d}}$. One can define $\mathrm{P}$ as the bounded intersection of affine half spaces. A polytope is rational if all vertices have rational coordinates. $\mathrm{P}^{\circ}$ denote the relative interior of $\mathrm{P}$. for a positive integer $t$, let $L_{P}(t)$ denote the number of integer points in the dilated polytope $\mathrm{tP}=\{\mathrm{tx}, \mathrm{x} \in \mathrm{P}\}$.
The fundamental result about the structure of $\mathrm{L}_{\mathrm{P}}(\mathrm{t})$ is given by theorems (3) and (4).

\section{Vector partition function:}

Let $\phi_{b}^{\circ}(b)$ count the integer solutions of $\mathrm{x}>0, \quad \mathrm{Ax}=\mathrm{b}, \quad \mathrm{A} \in \mathrm{M}_{\mathrm{mxd}}$ And $\mathrm{b} \in \mathrm{Z}^{\mathrm{m}}$. Both $\phi_{\mathrm{A}}(\mathrm{b})$ and $\phi_{\mathrm{b}}^{\circ}(\mathrm{b})$ are quasi-polynomials, and can hence be algebraically defined for arguments which are not integer vector in the positive span of $A$. the following identity shows the relationship between the two functions.

\section{Theorem 1, [2]:}

The quasi-polynomials $\phi_{\mathrm{A}}(\mathrm{b})$ and $\phi_{\mathrm{b}}^{\circ}(\mathrm{b})$ satisfy

$$
\phi_{\mathrm{A}}(-\mathrm{b})=(-1)^{\mathrm{d}-\operatorname{rank}(\mathrm{A})} \phi_{\mathrm{A}}^{\circ}(\mathrm{b})
$$

\section{Corollary 1, [2]:}

The quasi-polynomials $\phi_{\mathrm{A}}$ (b) satisfy

$$
\phi_{\mathrm{A}}(-\mathrm{b})=(-1)^{\mathrm{d}-\mathrm{rank}(\mathrm{A})} \phi_{\mathrm{A}}(-\mathrm{b}-\mathrm{r})
$$

\section{Lemma 1, [3]:}

Let $\Phi_{\mathrm{A}}(\mathrm{b})$ be a vector partition functions for the system $A x=b, A \in M_{m x d}$

And $\mathrm{b} \in \mathrm{Z}^{\mathrm{m}}$, then $\Phi_{\mathrm{A}}(\mathrm{b})$ equals the coefficients of $Z^{b}=z_{1}^{b_{1}}, \ldots, z_{m}^{b_{m}}$ of the function $\mathrm{f}(\mathrm{Z})=\frac{1}{\left(1-\mathrm{Z}^{\mathrm{c}_{1}}\right) \ldots\left(1-\mathrm{Z}^{\mathrm{c}_{\mathrm{d}}}\right)} \quad$ expanded as a power series centered at $Z=0$. Equivalently, the coefficients of $Z^{b}$ in $f(z)$ equals the constant 
term in $\frac{f(Z)}{Z^{b}}$ denoted by const $\frac{f(Z)}{Z^{b}}$, so Eulers lemma can be stated as:

$\phi_{\mathrm{A}}(\mathrm{b})=\mathrm{const} \frac{1}{\left(1-\mathrm{Z}^{\mathrm{c}_{1}}\right)\left(1-\mathrm{Z}^{\mathrm{c}_{2}}\right) \ldots\left(1-\mathrm{Z}^{\mathrm{c}_{\mathrm{d}}}\right) \mathrm{Z}^{\mathrm{b}}}$

In a series of articles $[4,5,6]$, complex integration of $\frac{\mathrm{f}(\mathrm{Z})}{\mathrm{Z}^{\mathrm{b}}}$ are used to compute $\Phi_{\mathrm{A}}(\mathrm{b})$ for special case of A.

Here we expand $\frac{f(Z)}{Z^{b}}$ into partial fractions to compute its constant term, and hence $\Phi_{\mathrm{A}}(\mathrm{b})$.

\section{The modified partial fraction method:}

This section illustrates the idea of our computation. Our goal is to derive, $\phi_{\mathrm{A}}(\mathrm{b})=\mathrm{const} \frac{1}{\left(1-Z^{\mathrm{c}_{1}}\right)\left(1-Z^{\mathrm{c}_{2}}\right) \ldots\left(1-Z^{\mathrm{c}_{\mathrm{d}}}\right) Z^{\mathrm{b}}}$.

We start by expanding

$\frac{1}{\left(1-Z^{c_{1}}\right)\left(1-Z^{c_{2}}\right) \ldots\left(1-Z^{c_{d}}\right) Z^{b}} \quad$ into partial fractions in one of the components of $Z$, say $\mathrm{z}_{1}$, therefore,

$\frac{1}{\left(1-Z^{c_{1}}\right)\left(1-Z^{c_{2}}\right) \ldots\left(1-Z^{c_{d}}\right) Z^{b}}=\frac{1}{Z_{2}^{b_{2}} \ldots Z_{m}^{b_{m}}} \sum_{k=1}^{d} \frac{A_{k}\left(Z, b_{1}\right)}{1-Z^{c_{k}}}+\sum_{j=1}^{b_{1}} \frac{B_{j}(Z)}{Z_{1}^{j}}$

Here $A_{k}$ and $B_{j}$ are polynomials in $z_{1}$, rational functions in $\mathrm{z}_{2}, \ldots, \mathrm{z}_{\mathrm{m}}$, and exponential in $b_{1}$. The two sums on the right- hand side correspond to the analytic and the meromorphic part with respect to $z_{1}=0$. The latter does not contribute to the $\mathrm{z}_{1}$-constant term, whence

$$
\begin{aligned}
\phi_{\mathrm{A}}(\mathrm{b}) & =\text { const }_{\mathrm{z}_{2} \ldots \mathrm{z}_{\mathrm{m}}}\left(\frac{1}{\mathrm{z}_{2}^{\mathrm{b}_{2}} \ldots \mathrm{z}_{\mathrm{m}}^{\mathrm{b}_{\mathrm{m}}}} \operatorname{const}_{\mathrm{z}_{1}}\left(\sum_{\mathrm{k}=1}^{\mathrm{d}} \frac{\mathrm{A}_{\mathrm{k}}\left(\mathrm{Z}, \mathrm{b}_{1}\right)}{1-\mathrm{Z}^{\mathrm{c}_{\mathrm{k}}}}\right)\right) \\
& =\operatorname{const}\left(\frac{1}{\mathrm{z}_{2}^{\mathrm{b}_{2}} \ldots \mathrm{z}_{\mathrm{m}}^{\mathrm{b}_{\mathrm{m}}}} \sum_{\mathrm{k}=1}^{\mathrm{d}} \mathrm{A}_{\mathrm{k}}\left(0, \mathrm{z}_{2}, \ldots, \mathrm{z}_{\mathrm{m}}, \mathrm{b}_{1}\right)\right)
\end{aligned}
$$

The effect of one partial fraction is to eliminate one of the variables of the generating function, at the cost of replacing one rational function by a sum of such. It is best to illustrate the above idea through an actual example.

\section{An illustrating example}

Consider the constraints

$$
\begin{aligned}
& \mathrm{x}_{1}+2 \mathrm{x}_{2}+=\mathrm{a} \\
& \mathrm{x}_{1}+\mathrm{x}_{2}+\mathrm{x}_{4}=\mathrm{b} \quad, \mathrm{x}_{1}, \mathrm{x}_{2}, \mathrm{x}_{3}, \mathrm{x}_{4} \geq 0
\end{aligned}
$$

This can be written as $A x=b$, where

$$
\mathrm{A}=\left(\begin{array}{llll}
1 & 2 & 1 & 0 \\
1 & 1 & 0 & 1
\end{array}\right) \text {, and } \mathrm{b}=(\mathrm{a}, \mathrm{b}) \text {, so }
$$

$\phi_{A}(a, b)$ counts the integer solutions of the above system, so by Eulers lemma,

$\phi_{\mathrm{A}}(\mathrm{a}, \mathrm{b})=\mathrm{const} \frac{1}{\left(1-\mathrm{z}_{1} \mathrm{z}_{2}\right)\left(1-\mathrm{z}_{1}^{2} \mathrm{z}_{2}\right)\left(1-\mathrm{z}_{1}\right)\left(1-\mathrm{z}_{2}\right) \mathrm{z}_{1}^{\mathrm{a}} \mathrm{z}_{2}^{\mathrm{b}}}$

We first expand into partial fractions with respect to $\mathrm{z}_{2}$

$$
\begin{aligned}
& \frac{1}{\left(1-z_{1} z_{2}\right)\left(1-z_{1}^{2} z_{2}\right)\left(1-z_{2}\right) z_{2}^{b}}= \\
& \frac{z_{1}^{b+1}}{\left(1-z_{1}\right)^{2}}+\frac{z_{1}^{2 b+3}}{1-z_{1} z_{2}}+\frac{\left(1-z_{1}\right)\left(1-z_{1}^{2}\right)}{1-z_{1}^{2} z_{2}}+\frac{\frac{1}{\left(1-z_{1}\right)\left(1-z_{1}^{2}\right)}}{1-z_{2}}+\sum_{k=1}^{b} \frac{\cdots}{z_{2}^{k}}
\end{aligned}
$$

Taking constant terms gives

$\phi_{\mathrm{A}}(\mathrm{a}, \mathrm{b})=\operatorname{const}_{\mathrm{z}_{1}}\left(\frac{1}{\left(1-\mathrm{z}_{1}\right) \mathrm{z}_{1}^{\mathrm{a}}} \operatorname{const}_{\mathrm{z}_{2}}\left(\frac{1}{\left(1-\mathrm{z}_{1} \mathrm{z}_{2}\right)\left(1-\mathrm{z}_{1}^{2} \mathrm{z}_{2}\right)\left(1-\mathrm{z}_{2}\right) \mathrm{z}_{2}^{\mathrm{b}}}\right)\right)$

$=$ const $\left(\frac{1}{\left(1-z_{1}\right) z_{1}^{a}}\left(-\frac{z_{1}^{b+1}}{\left(1-z_{1}\right)^{2}}+\frac{z_{1}^{2 b+3}}{\left(1-z_{1}\right)\left(1-z_{1}^{2}\right)}+\frac{1}{\left(1-z_{1}\right)\left(1-z_{1}^{2}\right)}\right)\right)$

$=$ const $\left(-\frac{z_{1}^{b-a+1}}{\left(1-z_{1}\right)^{3}}+\frac{z_{1}^{2 b-a+3}}{\left(1-z_{1}\right)^{2}\left(1-z_{1}^{2}\right)}+\frac{1}{\left(1-z_{1}\right)\left(1-z_{1}^{2}\right) z_{1}^{a}}\right)$

Our work is to find the constant term of the above expression with respect to one variable which is $z_{2}$, therefore we get,

$\phi_{A}(a, b)=$ const

$\left(\frac{1}{\left(1-z_{1}\right) z_{1}^{a}}\left(-\frac{z_{1}^{b+1}}{\left(1-z_{1}\right)^{2}}+\frac{z_{1}^{2 b+3}}{\left(1-z_{1}\right)\left(1-z_{1}^{2}\right)}+\frac{1}{\left(1-z_{1}\right)\left(1-z_{1}^{2}\right)}\right)\right)$

Others terms are eliminated since they contents some variables and we wants only constant term, therefore

const $\left(-\frac{z_{1}^{b-a+1}}{\left(1-z_{1}\right)^{3}}+\frac{z_{1}^{2 b-a+3}}{\left(1-z_{1}\right)^{2}\left(1-z_{1}^{2}\right)}+\frac{1}{\left(1-z_{1}\right)\left(1-z_{1}^{2}\right) z_{1}^{a}}\right)$ 
Now, each term of the three constant terms termed as counting integer solutions to linear systems.

$$
\text { const }\left(-\frac{\mathrm{z}_{1}^{\mathrm{b}-\mathrm{a}+1}}{\left(1-\mathrm{z}_{1}\right)^{3}}\right)=0 \text { if } \mathrm{b}-\mathrm{a}+1>0 .
$$

Equivalently $\mathrm{b} \geq \mathrm{a}$.

If $\mathrm{b}<\mathrm{a}$, we use Taylor's expansion of $\frac{1}{\left(1-z_{1}\right)^{3}}$ we get

$\frac{1}{\left(1-z_{1}\right)^{3}} \sum_{\mathrm{k} \geq 0}\left(\begin{array}{c}\mathrm{k}+2 \\ 2\end{array}\right) \mathrm{z}_{1}^{\mathrm{k}}$ This gives

const $\left(\frac{1}{\left(1-z_{1}\right)^{3} z_{1}^{a-b+1}}\right)=\left(\begin{array}{c}a-b+1 \\ 2\end{array}\right)=\frac{(a-b)^{2}}{2}+\frac{a-b}{2}$

Since we get constant term only if the power of $z_{1}$ is zero, that is $k=b-a+1$.

Therefore the first term is,

const $\left(\frac{1}{\left(1-z_{1}\right)^{3} z_{1}^{a-b+1}}\right)=\left\{\begin{array}{c}0 \quad \text { if } b \geq a \\ \frac{(a-b)^{2}}{2}+\frac{a-b}{2} \quad \text { if } b \leq a+1\end{array}\right.$

For the second term in (1), we have

const $\left(\frac{z_{1}^{2 b-a+3}}{\left(1-z_{1}\right)^{2}\left(1-z_{1}^{2}\right)}\right)=0$ if $2 b-a+2 \geq 0$

If $a \geq 2 b+3$, we expand into partial fractions again, in general

$\frac{1}{\left(1-z_{1}\right)^{2}\left(1-z_{1}^{2}\right)}=\frac{1}{\left(1-z_{1}\right)^{2}\left(1-z_{1}\right)\left(1+z_{1}\right)}=\frac{1}{\left(1-z_{1}\right)^{3}\left(1+z_{1}\right)}$

Taylor expansion for $\frac{1}{\mathrm{z}_{1}^{\mathrm{a}-2 \mathrm{~b}+3}}$ about $\mathrm{z}_{0}=1$ is founded, let $t=a-2 b+3$

$$
\begin{aligned}
& \frac{1}{z_{1}^{t}}=1+(-t)\left(z_{1}-1\right)+\frac{(-t)(-t-1)}{2 !}\left(z_{1}-1\right)^{2}+ \\
& \frac{(-t)(-t-1)(-t-2)}{3 !}\left(z_{1}-1\right)^{3}+\ldots
\end{aligned}
$$

Therefore

$$
\begin{aligned}
& \text { const }_{z_{1}} \frac{1}{\left(1-z_{1}\right)^{3}\left(1+z_{1}\right)} . \\
& \left(1+(-t)\left(z_{1}-1\right)+\frac{(-t)(-t-1)}{2 !}\left(z_{1}-1\right)^{2}+\frac{(-t)(-t-1)(-t-2)}{3 !}\left(z_{1}-1\right)^{3}+\ldots\right)
\end{aligned}
$$

By subtitled $\frac{1}{\left(1-z_{1}\right)^{3}\left(1+z_{1}\right)}=\frac{1 / 2}{\left(1-z_{1}\right)^{3}}+\frac{1 / 4}{\left(1-z_{1}\right)^{2}}+\frac{1 / 8}{\left(1-z_{1}\right)}+\frac{1 / 8}{\left(1+z_{1}\right)}$

$\frac{1}{\left(1-z_{1}\right)^{2}\left(1+z_{1}\right)}=\frac{1 / 2}{\left(1-z_{1}\right)^{2}}+\frac{1 / 4}{\left(1-z_{1}\right)}$

And

$\frac{1}{\left(1-z_{1}\right)\left(1+z_{1}\right)}=\frac{1 / 2}{\left(1-z_{1}\right)}+\frac{1 / 2}{\left(1+z_{1}\right)}$

Into (2), and sum the terms with similar denominator, we get

const $_{z_{1}}\left(\frac{1 / 2}{\left(1-z_{1}\right)^{3}}+\frac{1 / 4+t}{\left(1-z_{1}\right)^{2}}+\frac{1 / 8+t / 4+1 / 4(-t)(-t-1)}{\left(1-z_{1}\right)}+\right.$
$\left.\frac{1 / 8+t / 4+(-t)(-t-1) / 4}{4\left(1+z_{1}\right)}\right)$

By substation $t=2 b-a+3$, the constant is $\frac{(a-2 b)^{2}}{4}+\frac{2 b-a}{2}+\frac{1-(-1)^{a+1}}{8}$

Similarly to the first constant term computations, this identity is also valid for $a=2 b+2,2 b+1$ and $2 b$, hence

const $\left(\frac{z_{1}^{2 b-a+3}}{\left(1-z_{1}\right)^{2}\left(1-z_{1}^{2}\right)}\right)=$

$\left\{\begin{array}{cc}0 & \text { if } a \leq 2 b+2 \\ \frac{(a-2 b)^{2}}{4}+\frac{2 b-a}{2}+\frac{1-(-1)^{a+1}}{8} & \text { if } a \geq 2 b\end{array}\right.$

For the last term, the constant is

$$
\begin{aligned}
& \text { const }\left(\frac{1}{\left(1-z_{1}\right)\left(1-z_{1}^{2}\right) z_{1}^{a}}\right)= \\
& \text { const } z_{z_{1}}\left(\frac{1 / 2}{\left(1-z_{1}\right)^{3}}+\frac{1 / 4+a / 2}{\left(1-z_{1}\right)^{2}}+\frac{1 / 8+a^{2} / 4+a / 2}{\left(1-z_{1}\right)}+\frac{(-1)^{a} / 8}{\left(1+z_{1}\right)}\right) \\
& =\frac{\mathrm{a}^{2}}{4}+\mathrm{a}+\frac{7+(-1)^{\mathrm{a}}}{8}
\end{aligned}
$$

Summing up all terms in (1) gives:

$\phi_{A}(a, b)=$

$$
\left\{\begin{array}{cl}
\frac{a^{2}}{4}+a+\frac{7+(-1)^{a}}{8} & \text { if } a \leq b \\
a b-\frac{a^{2}}{4}-\frac{b^{2}}{4}+\frac{a+b}{2}+\frac{7+(-1)^{a}}{8} & \text { if } \frac{a}{2}-1 \leq b \leq a+1 \\
\frac{b^{2}}{2}+\frac{3 b}{2}+1 & \text { if } b \leq \frac{a}{2}
\end{array}\right.
$$

Also, we can show that, by corollary (1)

$$
\phi_{\mathrm{A}}(\mathrm{a}, \mathrm{b})=\phi_{\mathrm{A}}(-\mathrm{a}-4,-\mathrm{b}-3)
$$


We also modified the method of finding partial fraction using the following theorem which stat that:

\section{Theorem (2), [7, p.273]:}

If $a$ is a simple root of $Q(x)$ so that $\mathrm{Q}(\mathrm{x})=(\mathrm{x}-\mathrm{a}) \mathrm{Q} 1(\mathrm{x}), \mathrm{Q} 1(\mathrm{a}) \neq 0$, then the function $\frac{\mathrm{P}(\mathrm{x})}{\mathrm{Q}(\mathrm{x})}$ can be written in one and only one way in the form $\frac{P(x)}{Q(x)}=\frac{C}{x-a}+\frac{P 1(x)}{Q 1(x)}$ where $C$ is a constant. $\mathrm{C}$ can be calculated by using the form $C=\frac{P(a)}{Q_{1}(a)}=\frac{P(a)}{Q^{\prime}(x)}$.

The same example is solved using a modified method by assuming that

$$
\frac{P\left(z_{2}\right)}{Q\left(z_{2}\right)}=\frac{1}{\left(1-z_{1} z_{2}\right)\left(1-z_{1}^{2} z_{2}\right)\left(1-z_{2}\right) z_{2}^{b}}
$$

Write $\mathrm{Q}\left(\mathrm{z}_{2}\right)$ as

$$
\mathrm{Q}\left(\mathrm{z}_{2}\right)=\left(\mathrm{z}_{2}-\mathrm{a}_{1}\right)\left(\mathrm{z}_{2}-\mathrm{a}_{2}\right) \ldots\left(\mathrm{z}_{2}-\mathrm{a}_{\mathrm{k}}\right)
$$

therefore,

$$
\begin{aligned}
R(z) & =-z_{1}\left(z_{2}-\frac{1}{z_{1}}\right) \cdot z_{1}^{2}\left(z_{2}-\frac{1}{z_{1}^{2}}\right) \cdot\left(z_{2}-1\right) z_{2}^{b} \\
& =-z_{1}^{3}\left(z_{2}-\frac{1}{z_{1}}\right) \cdot\left(z_{2}-\frac{1}{z_{1}^{2}}\right) \cdot\left(z_{2}-1\right) z_{2}^{b}
\end{aligned}
$$

Then

$$
\mathrm{Q}\left(\mathrm{z}_{2}\right)=\left(\mathrm{z}_{2}-\frac{1}{\mathrm{z}_{1}}\right) \cdot\left(\mathrm{z}_{2}-\frac{1}{\mathrm{z}_{1}^{2}}\right) \cdot\left(\mathrm{z}_{2}-1\right) \mathrm{z}_{2}^{\mathrm{b}}
$$

and $\mathrm{P}\left(\mathrm{z}_{2}\right)=-\frac{1}{\mathrm{z}_{1}^{3}}$, with constant $\mathrm{C}_{\mathrm{k}}=\frac{\mathrm{P}\left(\mathrm{a}_{\mathrm{k}}\right)}{\mathrm{Q}^{\prime}\left(\mathrm{a}_{\mathrm{k}}\right)}$. Hence by computation we get,

$$
\begin{aligned}
& C_{1}=\frac{P\left(a_{1}\right)}{Q^{\prime}\left(a_{1}\right)}=-\frac{z_{1}^{b+1}}{\left(1-z_{1}\right)^{2}}, \\
& \mathrm{C}_{2}=\frac{\mathrm{P}\left(\mathrm{a}_{2}\right)}{\mathrm{Q}^{\prime}\left(\mathrm{a}_{2}\right)}=-\frac{\mathrm{z}_{1}^{3+2 \mathrm{~b}}}{\left(1-\mathrm{z}_{1}\right)\left(1-\mathrm{z}_{1}^{2}\right)}
\end{aligned}
$$

and

$$
\mathrm{C}_{3}=\frac{\mathrm{P}\left(\mathrm{a}_{3}\right)}{\mathrm{Q}^{\prime}\left(\mathrm{a}_{3}\right)}=-\frac{1}{\left(1-\mathrm{z}_{1}\right)\left(1-\mathrm{z}_{1}^{2}\right)}
$$

Those are the constant terms that are founded as before. All other constant can be found using the same way. This makes the solution easier.

\section{Theorem (3), [2]:}

If $\mathrm{P}$ is a convex rational polytope, then the functions $\quad \mathrm{L}_{\mathrm{P}}(\mathrm{t})$ and $\mathrm{L}_{\mathrm{P}}^{\circ}(\mathrm{t})$ are quasipolynomials in $t$ whose degree is the dimension $\mathrm{pf} P$. if $\mathrm{P}$ has integer vertices, then $\mathrm{L}_{\mathrm{P}}(\mathrm{t})$ and $\mathrm{L}_{\mathrm{P}}^{\circ}(\mathrm{t})$ are polynomials.

\section{Theorem (4), [2]:}

The quasi-polynomials $\mathrm{L}_{\mathrm{P}}(\mathrm{t})$ and $\mathrm{L}_{\mathrm{p}}^{\circ}(\mathrm{t})$ satisfy

$$
\mathrm{L}_{\mathrm{P}}(-\mathrm{t})=(-1)^{\mathrm{dimP}} \mathrm{L}_{\mathrm{P}}^{\circ}(\mathrm{t})
$$

Suppose the convex rational polytope $\mathrm{P} \subset \mathrm{R}^{\mathrm{d}}$ is given by an intersection of half spaces, that is $\mathrm{P}=\left\{\mathrm{x} \in \mathrm{R}^{\mathrm{d}}: \mathrm{Ax} \leq \mathrm{b}\right\}$, where $\mathrm{A} \in \mathrm{M}_{\mathrm{mxd}}$ and $\mathrm{b} \in \mathrm{Z}^{\mathrm{m}}$. We may convert these inequalities into equalities by introducing slack variables.

If $\mathrm{P}$ has rational vertices, we can choose $\mathrm{A}$ and $b$ in such a way that their entries are integer, without loss of generality nonnegative ones.

The connection to vector partition functions is now evident. Since $\mathrm{tP}=\left\{\mathrm{x} \in \mathrm{R}_{\geq 0}^{\mathrm{d}}: \mathrm{Ax} \leq \mathrm{tb}\right\}$, we obtain $L_{P}(t)=\phi_{A}(t b)$ as special evaluation of $\phi_{\mathrm{A}}(\mathrm{b})$ as an example, the quadrilateral $\mathrm{Q}$ described by

$$
\begin{aligned}
& x, y>0, \quad x+2 y \leq 5 \\
& x+y \leq 4
\end{aligned}
$$

As special case of the polygons appearing in section (4) with vertices $(0,0),(4,0),(3,1)$ and $(0,5 / 2)$ has the Ehrhart-quasi polynomial

$$
L_{Q}(t)=\phi_{A}(5 t, 4 t)=\frac{23}{4} t^{2}+\frac{9}{2} t+\frac{7+(-t)^{t}}{8}
$$

\section{References}

[1] J.Gubeladze, Course in information, Math 890, Discrete Geometry Fall (2003), math.sfsu.edu/gubeladze/fall2003/discrete. pdf-63k., (2003).

[2] R. P. Stanley, Enumerative combinatorics, Wadsworth \& Brooks/ Cole Advanced Books \& software, California, 1986.

[3] A. I.Barvinok, computing the Ehrhart polynomial of a convex lattice polytope, Discrete Comput. Geom. 12, n0. 1, (1994), 35-48 
[4] M. Beck, counting lattice points by means of the residue theorem, Ramanujan J. 4, (3), (2000), 299-310.

[5] M. Beck, R.Diaz, and S.Robins, the Frobenius problem, rational polytopes, and Fourier Dedekined sums, J. Number Theory 96, no. 1, (2002), 1-21.

[6] A.S. Shatha, on the volume and integral points of a polyhedron in $\mathfrak{R}^{\mathrm{n}}, \mathrm{Ph} . \mathrm{D}$ thesis, Al-Nahrain University, collage of science/ Mathematics and Computer application, 2005.

[7] L. R. Ford, SR., and L. R. Ford, SJ., Calculus, McGraw-Hill Book Company. Inc., 1963.

[8] M. Beck and D. Pixton, the Ehrhart Polynomial of the Brikhoff polytope, Discrete Comput. Geom. 30, no. 4, (2003), 623-637.

[9] M. Brion and M. Vergne, Residue formulae, vector partition functions and lattice points in rational polytopes, J. Amer. Math. Soc. 10, no. 4, (1997), 797833.

[10] S. E. Cappell and J. L Shaneson, EulerMaclaurin expansions for lattice above dimesion one, C. R. Acad. Sci. Paris S'er. I Math. 321, no. 7, (1995), 885-890.

[11] B. Chen, Lattice points, Dedekined sums, and Ehrhart polynomials of lattice polyhedra, Discrete Comput. Geom. 28, no. 2, (2002), 175-199.

[12] R. Diaz, and S. Robins, the Ehrhart polynomials of a lattice polytpe, Ann. of Math. (2) 145, no. 3, (1997), 503-518.

[13] J. E. Pommersheim, Toric varieties, lattice points and Dedekind sums, Math. Ann. 295, no. 1, (1993), 1-24.

[14] M. Beck, J. A. De. Loera, M. Develin, J. Peifle and R. P. Stanley, Coefficients and roots of Ehrhart polynomials, conference on integer points in Polyhedra (13-17) July in Snowbird, 2003), 1-24.

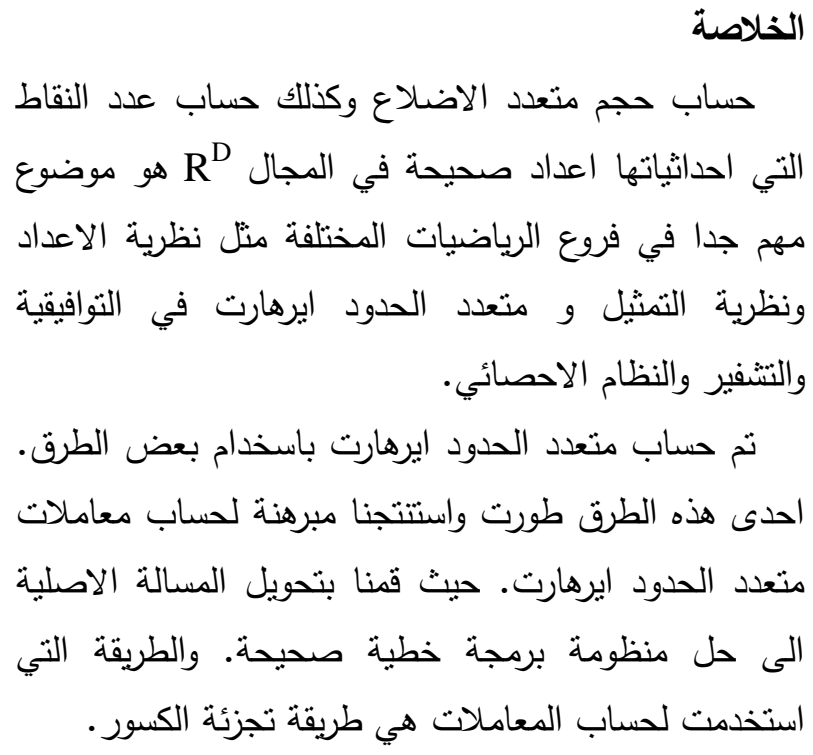

